\title{
Vom Rascheln der Blätter
}

Erhard Taverna

Auch das Schreiben kennt so etwas wie Jahreszeiten. Im Frühling keimen die Ideen, im Sommer reifen sie heran, und im Herbst flattern die publizierten Titel durch die Buchkataloge. Drei Mal ist Suspense angesagt. Einmal im Sprechzimmer, wenn der Aha-Effekt ausbleibt, einmal, wenn die Nachrichtenfülle überfordert, und einmal, wenn tüchtige Kollegen auf schiefe Wege geraten.

\section{Orthopädie für Hausärzte}

Probleme des Bewegungsapparates sind in der Allgemeinpraxis häufig. Genaue Zahlen gibt es dazu keine, in vielen Publikationen wird der Anteil auf 15 bis 50\% geschätzt. Die Abweichungen sind typisch für eine fachärztliche Ausrichtung, die über kein einheitliches Curriculum verfügt, so dass individuelle Neigung und Einzugsgebiet den Ausschlag geben. Unbestritten bleibt, dass orthopädische Probleme wichtig sind und jeder Hausarzt zumindest über ein Basiswissen verfügen sollte.

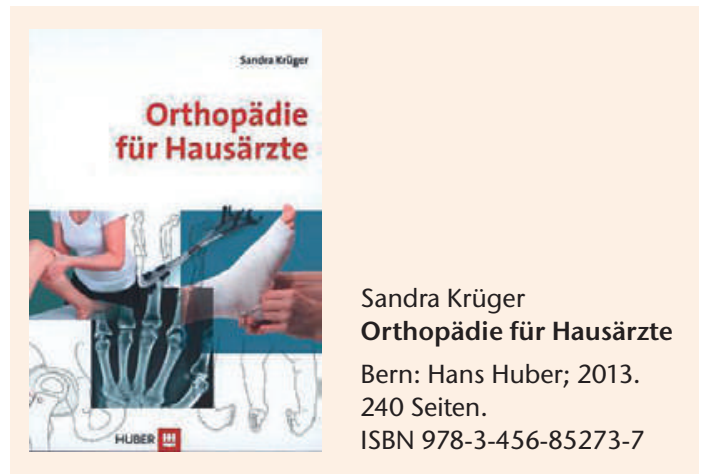

Dazu gibt es verdienstvolle Werke, wie das von Alfred M. Debrunner «Orthopädie - Orthopädische Chirurgie» (4. Auflage 2002) oder neu das Buch der Berliner Orthopädin Sandra Krüger, ebenfalls im Huber-

\section{Als praxisorientiertes, umfassendes Kompendium und bequemes Nachschlagewerk eignet sich das Buch in jedem Fall.}

Verlag herausgebracht. «Orthopädie für Hausärzte bietet eine Übersicht über die häufigsten Unfallverletzungen, degenerativen Krankheiten, Rückenbeschwerden und rheumatischen Krankheiten in der hausärztlichen Sprechstunde.
Der Schwerpunkt liegt in der klinischen Diagnostik. Die Autorin hebt besonders die präoperative Patienteninformation hervor, was eine enge Zusammenarbeit mit dem Spezialisten voraussetzt. Erkennen, beraten und rechtzeitig überweisen sind zentrale Anliegen, die in zehn Kapiteln, inklusive Kinderorthopädie, Rehabilitation und Nachbehandlung, kurz und klar abgehandelt werden. Jeder Arzt und jede Ärztin wird aus der breiten Palette an Behandlungsmöglichkeiten eine eigene Auswahl praktizieren. Als praxisorientiertes, umfassendes Kompendium und bequemes Nachschlagewerk eignet sich das Buch in jedem Fall.

\section{Gefühle machen Geschichte}

Historische Erzählungen bilden das menschliche Kollektivverhalten ab. Luc Ciompi, emeritierter Professor der Psychiatrie, und Elke Endert, Soziologin, thematisieren in ihrem gemeinsamen Buch «Gefühle machen Geschichte» die Wirkung kollektiver Emotionen auf das Denken und Handeln.

\section{Ein Buch, dessen lohnende}

\section{Lektüre die tägliche politische Informationsflut verständlicher macht.}

Das Konzept der Affektlogik, eine Theorie zu den Wechselwirkungen zwischen Fühlen und Denken, hat Luc Ciompi seit den Achzigerjahren, zunächst im sozialpsychiatrischen Kleinbereich, entwickelt und später verallgemeinert. Elke Endert baut auf die Emotionssoziologie von Norbert Elias auf und forscht über den Rechtsextremismus in Deutsch-

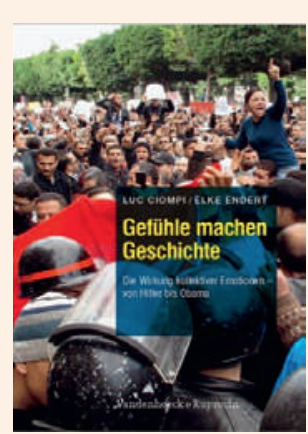

Luc Ciompi / Elke Endert Gefühle machen Geschichte. Die Wirkung kollektiver Emotionen von Hitler bis Obama

Göttingen: Vandenhoeck \& Ruprecht; 2011. 272 Seiten. ISBN 978-3-525-40436-2 
land. Beide betrachten ihr Buch «am ehesten als ausgedehnten, wissenschaftlichen Essay, der vielerlei bisher nicht als zusammengehörig angesehene Befunde unter einer einheitlichen Perspektive neu ordnet und vertieft».

Affektlogik wird als Lehre von den körperlichseelischen Reaktionen des Menschen auf lebenswichtige Situationen umschrieben. Wahrnehmung, Aufmerksamkeit, Gedächtnis und Denken führen zu gruppen- und kulturspezifischen Eigenwelten oder Mentalitäten, die von bestimmten Leitaffekten organisiert, bestätigt und gefestigt werden. Für diesen durchgehenden Konstruktivismus sprechen auch die Ergebnisse der modernen Hirnforschung. Gefühle sind unverzichtbare Instrumente zur Bewältigung von Wirklichkeit.

\section{Eine Abfolge von Komplikationen und überraschenden Ereignissen verdichtet den Plot und treibt ihn aktions- reich seinem dramatischen Höhepunkt entgegen.}

Der spannenden Einführung in die systemtheoretischen Grundlagen und soziologischen Emotionskonzepte folgen Analysen vergangener und gegenwärtiger Brennpunkte, wie des Nationalsozialismus, des Israel-Palästina-Konfliktes und der Kollision des Islam mit dem Westen. Kollektive Gefühle sind auch positiv gemeinschaftsbildend, dem «homo sapiens emotionalis» könnte vielleicht der angeborene Schönheitssinn weiterhelfen. Eine überraschende Aussage in dem an Einsichten und erhellendem Faktenwissen reichhaltigen Buch, dessen lohnende Lektüre die tägliche politische Informationsflut verständlicher macht.

\section{Vita minima}

Silvio Pacozzi, geboren 1962, ist Arzt für Innere Medizin in Brig. 1997 erschien im Rotten-Verlag der

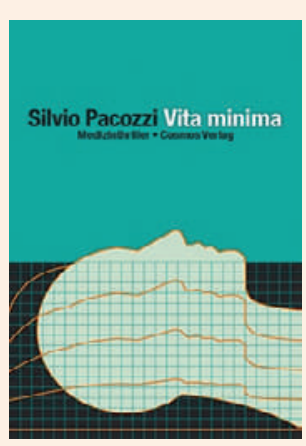

Silvio Pacozzi Vita minima Medizinthriller. Muri bei Bern: Cosmos; 2013. 281 Seiten ISBN 978-3-305-00441-6

Jugendkrimi «Die Dixie-Bande». Mit dem Debütroman «Vita minima» betritt der Autor die Thrillerbühne. Die Hauptfigur Manuel arbeitet als Kardiologe in einem Berner Privatspital. Präzise und detailliert werden Arbeitsmethoden und differentialdiagnostische Überlegungen (Anfängern und Laien ist der Pschyrembel zu empfehlen), Spitalabläufe, kollegiale oder weniger kollegiale Beziehungen informativ und einprägsam beschrieben.

Handwerkliches Können, kriminelles Profitstreben und vielschichtige Emotionen strapazieren das lädierte Familienleben des Protagonisten. Allmählich geht aus dem medizinischen Milieu der Keim der Geschichte auf. Eine Abfolge von Komplikationen und überraschenden Ereignissen verdichtet den Plot und treibt ihn aktionsreich seinem dramatischen Höhepunkt entgegen. In den Kühlkammern einer gutversteckten Intensivstation erfahren Leser und Leserinnen, was es mit dem Ausdruck «Vita minima» auf sich hat. Übel erpresst und roher Gewalt ausgesetzt, hat Dr. med. Manuel Capri grösste Mühe, aus dem Schlamassel wieder herauszufinden. Das Ende sei natürlich nicht verraten, nur so viel: Ein Freund wird geopfert, doch dem Kuss einer attraktiven Sekretärin und weiteren erotischen Angeboten kann unser Held knapp widerstehen. Die pubertierende Tochter findet zu Papa zurück, und der Bruder .... Lesen Sie doch selbst! 